\title{
ISOMETRIES OF $H^{p}$ SPACES OF THE TORUS
}

\author{
NAND LAL ${ }^{1}$ AND SAMUEL MERRILL III $^{2}$
}

\begin{abstract}
Denote by $\boldsymbol{H}^{p}(1 \leqq p \leqq \infty)$ the Banach spaces of complex-valued functions in $L^{p}$ of the torus whose Fourier coefficients vanish off a half plane determined by a lexicographic ordering. The, surjective isometries of the spaces $\boldsymbol{H}^{p}(p \neq 2)$ are characterized in terms of unimodular functions on the circle and conformal maps of the disc. For $1<p<\infty(p \neq 2)$ the proof depends upon a characterization of certain invariant subspaces previously given by the authors.
\end{abstract}

Let $A$ be the algebra of continuous complex-valued functions on $\{\lambda \in C:|\lambda|=1\}$ which are uniform limits of polynomials in $\lambda$. Denote by $H^{p}(d \theta)$ the closure of $A$ in $L^{p}(d \theta)$ where $d \theta$ denotes normalized Lebesgue measure on the circle (norm closure for $1 \leqq p<\infty ; w^{*}$ closure for $p=\infty$ ). It is well known that the Banach spaces $H^{p}(d \theta)$ may be identified with the Hardy classes by associating with each function in $H^{p}(d \theta)$ its analytic extension to the open unit disc via the Poisson integral.

DeLeeuw, Rudin, and Wermer [1], and independently Nagasawa [6], characterized the surjective isometries of $H^{\infty}(d \theta)$ and $H^{1}(d \theta)$. Forelli [2] extended the characterization to $H^{p}(d \theta)$ for $1<p<\infty, p \neq 2$. We state their results in Propositions 1 and 2.

Proposition 1. A linear operator $T$ of $H^{\infty}(d \theta)$ onto $H^{\infty}(d \theta)$ is an isometry if and only if

$$
(T f)(\lambda)=\alpha f(\tau(\lambda)) \quad\left(f \in H^{\infty}(d \theta) ;|\lambda|=1\right),
$$

where $\alpha$ is a complex constant of modulus 1 and $\tau$ is a conformal map of the unit disc onto itself.

Presented to the Society, January 25,1969 , under the title, Some results on generalized $H^{p}$ spaces; received by the editors March 29, 1971.

AMS 1970 subject classifications. Primary 46E15, 46J15; Secondary 30A98, 43A15.

Key words and phrases. $\boldsymbol{H}^{p}$ spaces, isometries, torus, invariant subspace, conformal map, logmodular algebra, double Fourier coefficients.

${ }^{1}$ This paper includes portions of the first author's doctoral thesis completed at the University of Rochester.

${ }^{2}$ Partially supported by NSF Grant GP 22713 at the University of Rochester.

(c) American Mathematical Society 1972 
Proposition 2. Let $1 \leqq p<\infty, p \neq 2$. A linear operator $T$ of $H^{p}(d \theta)$ onto $H^{p}(d \theta)$ is an isometry if and only if

$$
(T f)(\lambda)=\alpha\left(\tau^{\prime}(\lambda)\right)^{1 / p} f(\tau(\lambda)) \quad\left(f \in H^{p}(d \theta) ;|\lambda|=1\right),
$$

where $\alpha$ and $\tau$ are as in Proposition 1.

We denote by $A\left(T^{2}\right)$ the algebra of continuous, complex-valued functions on the torus $T^{2}=\{(z, w):|z|=|w|=1\}$ which are uniform limits of polynomials in $z^{m} w^{n}$ where $(m, n) \in \mathscr{S}=\{(m, n): n>0\} \cup\{(m, 0): m \geqq 0\}$. Denoting normalized Haar measure on $T^{2}$ by $d m$, we define $\boldsymbol{H}^{p}$ as the closure of $A\left(T^{2}\right)$ in $L^{p}(d m)$ (norm closure for $1 \leqq p<\infty ; w^{*}$ closure for $p=\infty$ ). The purpose of this paper is to present characterizations of the isometries of $\boldsymbol{H}^{p}$ onto $\boldsymbol{H}^{p}$ for $1 \leqq p \leqq \infty, p \neq 2$.

$\boldsymbol{H}^{p}$ consists of those functions in $L^{p}(\mathrm{dm})$ whose double Fourier coefficients vanish off the half-plane $\mathscr{S}$ which determines a lexicographic ordering. The maximal ideal space of $A\left(T^{2}\right)$ can be identified with $(\{z:|z|=1\} \times\{w:|w| \leqq 1\}) \cup(\{z:|z| \leqq 1\} \times\{0\})$, with $d m$ identified with $(z, w)=(0,0)$. Since $A(T)^{2}$ is a logmodular algebra we have at our disposal the results of [4].

We denote by $Z$ and $W$ the functions $Z(z, w)=z$ and $W(z, w)=w$. The closure in $L^{p}(d m)$ of the polynomials in $Z$ is denoted by $Z^{p}$; the closure of the polynomials in $Z^{m} W^{n}, n \geqq 1$, by $I^{p}$; and finally the closure of the polynomials in $Z$ and $Z$ by $L^{p}$. By [5, Lemma 5, p. 467],

$$
\boldsymbol{H}^{p}=Z^{p} \oplus I^{p}
$$

for $1 \leqq p \leqq \infty$, where $\oplus$ denotes algebraic direct sum. A function $f$ in $\boldsymbol{H}^{p}$ is inner if $|f| \equiv 1 ; f$ is outer if $f \cdot A\left(T^{2}\right)$ is dense in $\boldsymbol{H}^{p}$.

THEOREM 1. A linear operator $T$ of $\boldsymbol{H}^{\infty}$ onto $\boldsymbol{H}^{\infty}$ is an isometry if and only if

$$
(T f)(z, w)=\alpha f(\tau(z), w \sigma(z)) \quad\left(f \in \boldsymbol{H}^{\infty} ;|z|=|w|=1\right),
$$

where $\alpha$ is a complex constant of modulus $1, \tau$ is a conformal map of the unit disc onto itself, and $\sigma$ is a unimodular measurable function.

According to [1, Theorem 3, p. 695] it suffices to prove

THEOREM 2. A linear operator $\Psi$ of $\boldsymbol{H}^{\infty}$ onto $\boldsymbol{H}^{\infty}$ is an algebra automorphism if and only if

$$
(\Psi f)(z, w)=f(\tau(z), w \sigma(z)) \quad\left(f \in \boldsymbol{H}^{\infty} ;|z|=|w|=1\right),
$$

where $\tau$ and $\sigma$ are as in Theorem 1. 
LEMMA 1. If $\Psi$ is an algebra automorphism of $\boldsymbol{H}^{\infty}$, then $\Psi$ carries inner functions to inner functions, $\Psi Z^{\infty}=Z^{\infty}$, and $\Psi I^{\infty}=I^{\infty}$.

Proof. If $F$ is inner but $\Psi F$ is not, then there exists $\varepsilon>0$ such that $m(K)>0$ where $K=\{x:|\Psi F(x)|<1-\varepsilon\}$. Choose $h \in \boldsymbol{H}^{\infty}$ with $|h(x)|=1$ on $K$ and $|h(x)|=1-\varepsilon$ on $T^{2} \mid K$ [4, Theorem 5.9, p. 297]. If $\Psi g=h,\|F g\|_{\infty}=1$ but $\|\Psi(F g)\|_{\infty}=\|(\Psi F) h\|_{\infty} \leqq 1-\varepsilon$. Thus $\Psi F$ is inner.

Let $M$ be the closure of $\Psi I^{\infty}$ in $L^{2}(d m) . M$ is clearly invariant under multiplication by functions in $\boldsymbol{H}^{\infty}$ and also by $\bar{V}$ where $V=\Psi Z$. For if $f \in \boldsymbol{I}^{\infty}, f Z \in \boldsymbol{I}^{\infty}$, so $\Psi f=\Psi(f Z) \Psi(Z)$ or $\Psi(f Z)=(\Psi f)(\bar{V})$.

If $M$ has the form $F H^{2}$ for some inner function $F$, then $F \cdot \bar{V} \in M=$ $F H^{2}$ so $\bar{V} \in \boldsymbol{H}^{2}$. But $\Psi Z \in \boldsymbol{H}^{2}$ so it is a constant. This contradicts the fact that $\Psi$ is injective, so $M \subseteq I^{2}\left[4\right.$, p. 293]. It follows, using (3), that $\Psi I^{\infty} \subseteq I^{\infty}$. Applying the same argument to the automorphism $\Psi^{-1}$, we conclude that $\Psi^{\infty} I^{\infty}=I^{\infty}$.

To show that $\Psi Z^{\infty}=Z^{\infty}$, it suffices to show that $\Psi Z \in Z^{\infty}$. Write $f=\Psi Z$ and suppose $f=f_{1}+f_{2}$ where $f_{1} \in Z^{\infty}$ and $f_{2} \in I^{\infty}$. Then $f I^{\infty}=\Psi\left(Z I^{\infty}\right)=I^{\infty}$, so $f_{2}=f g$ for some $g \in I^{\infty}$. Thus $g=f_{2} \bar{f}=\left(f-f_{1}\right) \bar{f}=1-f_{1} \bar{f}$, which is orthogonal to $I^{\infty}$. Thus $g$ and hence $f_{2}$ vanish.

LEMMA 2. If $E_{1} \in Z^{\infty}$ and $E_{2} \in I^{\infty}$ are inner functions, and if for each Borel set $Y \subseteq T^{2}, \mu(Y)=m(X)$ where $X=\left\{(z, w):\left(E_{1}(z), E_{2}(z, w)\right) \in Y\right\}$, then $\mu \ll m$.

Proof. The Fourier-Stieltjes coefficients of $\mu$ are $\hat{\mu}(m, n)=\int \bar{E}_{1}^{m} \bar{E}_{2}^{n} d m$. Thus $\hat{\mu}(m, 0)=a^{m}$ for $m \geqq 0\left(a=\int \bar{E}_{1} d m\right)$. Since $E_{2} \in I^{\infty}, \hat{\mu}(m, n)=0$ for $n \geqq 1$. It follows that $\mu$ is the product measure $\mu=P d z \times d w=Q d m$, where $P(z)=\left(1-|a|^{2}\right) /|1-a z|^{2}, d z$ and $d w$ are each Lebesgue measure, and $Q \in L^{\infty}(d m)$. In particular, if $Y$ is $m$-null, then $X$ is $m$-null. This argument is based on Forelli [2, p. 724].

Proof of Theorem 2. By Lemma $1, \Psi W \in I^{\infty}$. In fact $\Psi W=W \sigma$ for some $\sigma(z)=\sigma(z, w) \in \boldsymbol{L}^{\infty}$, as can be shown by an argument similar to that by which we showed $\Psi Z \in Z^{\infty}$. Writing $\tau(z)=\tau(z, w)=(\Psi Z)(z, w)$, we see that $\tau$ is a conformal map of the disc by Proposition 1. Setting $E_{1}=\tau$ and $E_{2}=w \sigma$ in Lemma 2, we conclude that $f(\tau, w \sigma)$ is well defined for all measurable functions $f$. Thus (5) holds for all $f$ in the algebra $\mathscr{A}$ generated by $Z^{m} W^{n},(m, n) \in \mathscr{S}$.

To establish (5) for all $f \in \boldsymbol{H}^{\infty}$, it suffices to show that the automorphism $\Phi f=\Psi^{-1}(f(\tau, w \sigma))$ is the identity. We have seen that $\Phi Z=Z$ and $\Phi W=W$ and the proof of Proposition 1 shows that $\Phi$ is the identity on $Z^{\infty}$. Thus it suffices to show that $\Phi$ is the identity on $I^{\infty}$.

First we show that $\Phi\left(\chi_{K} W\right)=\chi_{K} W$ where $\chi_{K}$ is a charactersitic function in $L^{\infty}$. Since the function $\Phi\left(\chi_{K} W\right) / W$ is equal to its own square, it too is a characteristic function $\chi_{K^{\prime}} \in \boldsymbol{L}^{\infty}$. There remains only to show that $K=K^{\prime}$, 
or in fact that $K \subseteq K^{\prime}$ since the argument also applies to $\Phi^{-1}$. If not, there exists a nonzero continuous function $f \in \boldsymbol{L}^{\infty}$ with zero set $K_{1} \subseteq K / K^{\prime}$ of positive measure. Then

$$
0=\Phi(f W) \Phi\left(\chi_{K_{1}} W\right)=f W \chi_{K_{1}^{\prime}} W .
$$

Since $K_{1} \subseteq K, K_{1}^{\prime} \subseteq K^{\prime}$, so $f$ does not vanish on $K_{1}^{\prime}$. This contradicts (6).

Thus $\Phi\left(\chi_{K} W\right)=\chi_{K} W$, and in general for $g \in \boldsymbol{L}^{\infty}, \Phi\left(g W^{n}\right)=g W^{n}$ $(n \geqq 1)$. If $g \in \boldsymbol{I}^{\infty}, g=\sum_{i=1}^{n} g_{i} W^{i}+h W^{n}$ where $g_{i} \in \boldsymbol{L}^{\infty}$ and $h \in \boldsymbol{I}^{\infty}$. Since $\Phi g=$ $\sum_{i=1}^{n} g_{i} W^{i}+(\Phi h) W^{n}$ where $\Phi h \in I^{\infty}$, the Fourier coefficients of $g$ and $\Phi g$ agree, so $\Phi g=g$.

REMARK. Using essentially the same argument we can show that the automorphisms of $A\left(T^{2}\right)$ are also given by (5) except that here $\sigma$ is continuous. However, this can more easily be done by considering the homeomorphisms of the maximal ideal space of $A\left(T^{2}\right)$ induced by the automorphisms of the algebra.

THEOREM 3. A linear operator $T$ of $\boldsymbol{H}^{p}$ onto $\boldsymbol{H}^{p}(1 \leqq p<\infty, p \neq 2)$ is an isometry if and only if

$$
(T f)(z, w)=\alpha\left(\tau^{\prime}(z)\right)^{1 / p} \cdot f(\tau(z), w \sigma(z)),
$$

for all $f \in \boldsymbol{H}^{p}$, where $|z|=|w|=1, \alpha$ is a complex constant of modulus $1, \tau$ is a conformal map of the unit disc onto itself, and $\sigma$ is a unimodular measurable function on the circle.

The proof depends on our results in [5] on the characterization of sesqui-invariant subspaces of $\boldsymbol{H}^{p}$. A closed subspace $M \subseteq \boldsymbol{H}^{p}$ is called invariant if $f M \subseteq M$ for all $f \in \boldsymbol{H}^{\infty}$. An invariant subspace $M \subseteq \boldsymbol{H}^{p}$ is called sesqui-invariant if $Z M \subseteq M$ and simply invariant if this is not the case. If $M$ is sesqui-invariant, it has the form

$$
M=\chi_{E} \cdot \psi \cdot I^{p}
$$

where $\psi$ is unimodular and $\chi_{E}$ is the characteristic function of the support set of $M$ ([5, Theorem 3, p. 471]; see also [5, p. 473 for the torus case]). If $M$ is simply invariant, it has the form $M=\psi \boldsymbol{H}^{p}$ ( $\psi$ unimodular) by the generalized Beurling theorem [8].

Lemma 3. Let $F=T(1)$ and $E$ be the support set of $F$. Then $m(E)=1$.

Proof. Since $F \in H^{p}, \chi_{E}$ is independent of $w$, so $G=w\left(1-\chi_{E}\right) \in H^{p}$. Let $g=T^{-1}(G)$. Thus

$$
\begin{aligned}
\int|1 \pm g|^{p} d m & =\int|F \pm G|^{p} d m \\
& =\int|F|^{p} d m+\int|G|^{p} d m=1+\int|g|^{p} d m .
\end{aligned}
$$


Therefore

$$
\int|1+g|^{p} d m+\int|1-g|^{p} d m=2\left[1+\int|g|^{p} d m\right] .
$$

By $[7$, p. 275], $g=0$ a.e., so $m(E)=1$.

Proof of Theorem 3. Lemma 3 insures that $d v=|F|^{p} d m$ and $d m$ are mutually absolutely continuous. Using Forelli's argument [2, Proposition 2, p. 723] it follows that $S f=T f / F$ defines an isometry $S$ of $\boldsymbol{H}^{p}$ into $L^{p}(d v)$ which takes the algebra $\mathscr{A}$ generated by $Z^{m} W^{n},(m, n) \in \mathscr{S}$, multiplicatively into $L^{\infty}(d v)$. Write $E_{1}=S(Z)$ and $E_{2}=S(W)$. For $f \in \mathscr{A}$, we have

$$
T f(z, w)=F \cdot f\left(E_{1}, E_{2}\right) .
$$

We show that $E_{1} \in \boldsymbol{Z}^{\infty}$ and $E_{2} \in \boldsymbol{I}^{\infty}$. Since $F \in \boldsymbol{H}^{p}$, the sesqui-invariant subspace generated by $F$ has the form $J I^{p}$, where $J$ is unimodular. Thus $F=J G$ where $G \in I^{p}$ and the sesqui-invariant subspace generated by $G$ is $\boldsymbol{I}^{p}$. For $f \in S(\mathscr{A}), W F f \in I^{p}$, and the property of $G$ insures that $W^{2} J f \in I^{p}$. Thus the invariant subspace generated by $S(\mathscr{A})$ has the form $\psi I^{p}$ or $\psi \boldsymbol{H}^{p}, \psi$ unimodular.

In the first case $f \psi W \in \psi I^{p}$, so $f \in \boldsymbol{L}^{p} \oplus \boldsymbol{I}^{p}$ for all $f \in S(\mathscr{A})$ and similarly for the second case. In particular $E_{1}$ and $E_{2} \in \boldsymbol{L}^{\infty} \oplus \boldsymbol{I}^{\infty}$. The same argument applied to the algebra generated by $Z^{m} W^{n}, n \geqq 1$, shows that $\bar{E}_{\mathbf{1}} \in \boldsymbol{L}^{\infty} \oplus \boldsymbol{I}^{\infty}$, so $E_{1} \in \boldsymbol{L}^{\infty}$.

We conclude that $F \notin I^{p}$ (otherwise $T$ would map $\boldsymbol{H}^{p}$ onto $I^{p}$ ). Thus $\int \log |F| d m>-\infty$, so $F=J G$ where now $J$ is inner and $G$ is outer. Also $\left\{F E_{1}^{m}\right\}, m \geqq 0$, generate a simply invariant subspace, so by the usual argument $E_{1} \in Z^{\infty}$. Since $G$ is outer, the invariant subspace $N$ generated by $\left\{J E_{1}^{m} E_{2}^{n}\right\}, n>0$, is contained in $\boldsymbol{H}^{p}$. Since $N=\psi \boldsymbol{H}^{p}$ would imply that $\bar{E}_{1} \in \boldsymbol{H}^{p}$, we have $N=\psi I^{p}$. Thus $N \subseteq I^{p}$ so $J E_{2} \in I^{p} . J \in H^{p}$ but $J \notin I^{p}$ (because $F \notin I^{p}$ ), so $E_{2} \in I^{\infty}$. Thus $T$ takes $\boldsymbol{I}^{p}$ into $\boldsymbol{I}^{p}$.

Thus using Lemma $2, f\left(E_{1}, E_{2}\right)$ is well defined for all measurable functions $f$. The density of $\mathscr{A}$ in $\boldsymbol{H}^{p}, 1 \leqq p<\infty$, insures that (8) holds for all $f \in \boldsymbol{H}^{p}$. Imitating Forelli's argument $[2$, p. 726$]$ one shows that the function $Q$ constructed in the proof of Lemma 2 satisfies

$$
\int_{X}|F|^{p} d m=\int_{X} 1 / Q\left(E_{1}\right) d m
$$

for all Borel sets $X \subseteq T^{2}$. Since $T$ is surjective, both $T$ and $T^{-1}$ carry $I^{p}$ into $I^{p}$, so that $T I^{p}=I^{p}$. Again using the argument of [2] (beginning at the bottom of p. 726) it follows that $E_{1}$, considered as a function of $z$ alone, is a.e. the boundary value function of a conformal map $\tau_{1}$ of the disc onto itself. Define $\tau(z, w)=\tau_{1}(z)$. We have $\left|\tau^{\prime}\right|=1 / Q(\tau)$ and (9) becomes 


$$
\int_{X}|F|^{p} d m=\int_{X}\left|\tau^{\prime}\right| d m
$$

for all Borel sets $X \subseteq T^{2}$. Thus $F$ and $\left(\tau^{\prime}\right)^{1 / p}$ have the same modulus. Since the latter is outer, we can show that they differ by a constant of modulus one by showing $F$ is outer. If $F=J G, J$ inner and $G$ outer, then $G \boldsymbol{H}^{p}=$ $\boldsymbol{H}^{p}=T \boldsymbol{H}^{p}=F \boldsymbol{H}^{p}$. Dividing by $G, \boldsymbol{H}^{p}=J \boldsymbol{H}^{p}$, so $J \equiv \alpha,|\alpha|=1$.

To complete the proof it suffices to show that $E_{2}=W \sigma$ where $\sigma \in \boldsymbol{L}^{\infty}$. For this we need to show that $\sigma I^{p}=I^{p}$ (see the analogous argument for $p=\infty)$. But since $F$ and $1 / F$ are bounded, $\sigma I^{p}=\bar{W}(S W)\left(S I^{p}\right)=$ $\bar{W} S\left(W \boldsymbol{I}^{p}\right)=\bar{W} W \boldsymbol{I}^{p}=\boldsymbol{I}^{p}$.

For the case $p=1$, Theorem 3 can also be obtained by adapting the original argument of deLeeuw, Rudin and Wermer [1, Theorem 2, p. 694] in which they deduce the isometries of $H^{1}(d \theta)$ by exploiting the special properties of the extreme points of the unit ball of $H^{1}(d \theta)$. To do this one needs three facts about functions on the torus: (a) the extreme points of the unit ball of $\boldsymbol{H}^{\mathbf{1}}$ are the outer functions of norm one (Gamelin [3]), (b) the identity $\int f d m=\int f(\tau, w \sigma) \tau^{\prime} d m$ (a straightforward calculation), and (c) the result of Lemma 4 below. Let $B^{e}$ be the set of extreme points in the unit ball of $\boldsymbol{H}^{\mathbf{1}}, P(m)=\{z:|z|<1\} \times\{0\}$, and $D_{z}=\{z\} \times\{w:|w|<1\}$ for each $|z|=1$.

LEMMA 4. A function $f \in \boldsymbol{H}^{1}$ of norm 1 lies in the closure of $B^{e}$ if and only if (10) $\hat{f}$ has no zeros on $P(m)$ and $\hat{f}$ has no zeros on $D_{z}$ for almost all $z$.

Proof. If $f$ lies in the closure of $B^{e}$, then there exist $f_{n} \in B^{e}$ converging uniformly on compact sets to $\hat{f}$ on $P(m)$ and on each $D_{z}$ for almost all $z$. (10) follows.

Conversely suppose (10) holds. Define $f_{r}(z, w)=f(z, r w), 0<r<1$. Let $f_{r}=F_{r} g_{r}, F_{r}$ inner, $g_{r}$ outer. One shows that $F_{r}$ is independent of $r$, say $F_{r}=$ $F \in Z^{\infty}$. Let $h_{r}(z, w)=F(r z)$. Then $f$ is the $L^{1}$ limit of the outer functions $h_{r} g_{r}$, so $f$ lies in the closure of $B^{e}$.

\section{REFERENCES}

1. K. deLeeuw, W. Rudin and J. Wermer, The isometries of some function spaces, Proc. Amer. Math. Soc. 11 (1960), 694-698. MR 22 \#12380.

2. F. Forelli, The isometries of $H^{p}$, Canad. J. Math. 16 (1964), 721-728. MR 29 \#6336.

3. T. Gamelin, $H^{p}$ spaces and extremal functions in $H^{1}$, Trans. Amer. Math. Soc. 124 (1966), 158-167. MR 35 \#4731.

4. K. Hoffman, Analytic functions and logmodular Banach algebras, Acta Math. 108 (1962), 271-317. MR 26 \#6820.

5. S. Merrill and N. Lal, Characterization of certain invariant subspaces of $H^{p}$ and $L^{p}$ spaces derived from logmodular algebras, Pacific J. Math. 30 (1969), 463-474. MR 40 \#1778. 
6. M. Nagasawa, Isomorphisms between commutative Banach algebras with an application to rings of analytic functions, Kōdai Math. Sem. Rep. 11 (1959), 182-188. MR 22 \#12379.

7. H. Royden, Real analysis, Macmillan, New York, 1963. MR 27 \#1540.

8. T. P. Srinivasan, Simply invariant subspaces and generalized analytic functions, Proc. Amer. Math. Soc. 16 (1965), 813-818. MR 34 \#8219.

Department of Mathematics, North Texas State University, Denton, Texas 76201

Department of Mathematics, University of Rochester, Rochester, New York 14627 